\title{
OPEN Reinforcement learning derived chemotherapeutic schedules for robust patient-specific therapy
}

\author{
Brydon Eastman $\bowtie$, Michelle Przedborski \& Mohammad Kohandel
}

The in-silico development of a chemotherapeutic dosing schedule for treating cancer relies upon a parameterization of a particular tumour growth model to describe the dynamics of the cancer in response to the dose of the drug. In practice, it is often prohibitively difficult to ensure the validity of patient-specific parameterizations of these models for any particular patient. As a result, sensitivities to these particular parameters can result in therapeutic dosing schedules that are optimal in principle not performing well on particular patients. In this study, we demonstrate that chemotherapeutic dosing strategies learned via reinforcement learning methods are more robust to perturbations in patient-specific parameter values than those learned via classical optimal control methods. By training a reinforcement learning agent on mean-value parameters and allowing the agent periodic access to a more easily measurable metric, relative bone marrow density, for the purpose of optimizing dose schedule while reducing drug toxicity, we are able to develop drug dosing schedules that outperform schedules learned via classical optimal control methods, even when such methods are allowed to leverage the same bone marrow measurements.

In mathematical models of cancer treatment, one is often concerned with finding a chemotherapeutic dosing schedule that is optimal in some capacity ${ }^{1-3}$. Each different model allows distinct forms of this optimality metric. This shaping of optimality metric will often involve a trade-off of some variety: incredibly high doses of a potent chemotherapeutic can certainly annihilate the cancerous cells in tissue but in so doing will largely cause a great deal of harm to the patient. Modelers, then, are concerned with mathematically formulating this optimality metric in a way that preserves the health and longevity of their patient (virtual or otherwise). For instance, in Ref. ${ }^{2}$ the authors were concerned with maximizing the reduction in the total number of cancerous cells with the minimal total chemotherapeutic dose. They achieved this control by sampling 200 virtual patients from a particular parameter distribution, training 50 different reinforcement learning agents on differential equations representing the tumour growth of these patients, and applying these agents to these patients. In contrast, in Ref. ${ }^{1}$ the authors concerned themselves with maximizing the chemotherapeutic dose while minimizing the damage to healthy, proxy cells in the bone marrow. Practically, these are two (similar and related) methods for achieving the same ends, but the particulars of their formalization can lead to drastically different qualitative results.

In any situation, the models used to represent the delivery of the chemotherapeutic and the associated reduction in cancer cells can inform the choice of optimality metric. So too can the choice of model inform the method by which a modeller can find such an optimal dosing schedule of a chemotherapeutic. One such method, as employed in Ref. ${ }^{1}$, is that of optimal control theory. When the model that governs the behaviour we are trying to assert some control over is codified by differential equations, optimal control theory can provide a methodology for finding the dose delivery function that maximizes whatever optimality metric the modeller chooses (if such a metric has a maximum $)^{4}$. In some situations, this optimal control can be accomplished analytically as in Ref. ${ }^{1}$, in others (such as the objective functional presented in Eq. (3)) numerical techniques such as those employed by the GEKKO package may be required ${ }^{5}$.

A reinforcement learning approach can also be employed to maximize a given optimality metric (see, for instance, Ref. ${ }^{2}$ ). In a reinforcement learning context, when the state of the model at a given time $t$ is known, one can construct a controller function via the learned optimal policy. In contrast to optimal control, reinforcement learning more easily lends itself to situations where the model behaviour is governed by systems more complicated than just those that can be represented with differential equations (for instance, reinforcement learning has had great success in solving Atari games, arcade games, Backgammon, etc. ${ }^{6-8}$ ). In particular, as illustrated in Fig. 1, a reinforcement learner need only be provided with the action space of the environment; all other details 


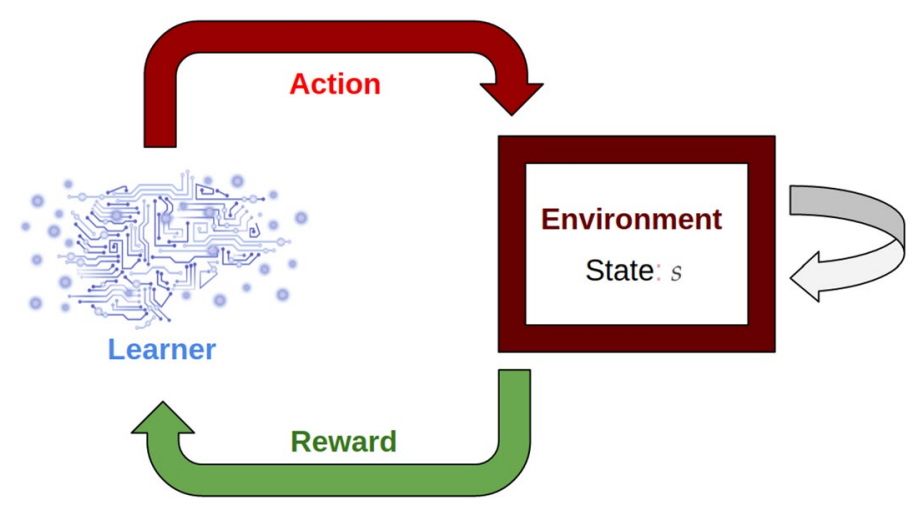

Figure 1. A reinforcement learning agent interacts with an environment as if the environment were a blackbox. This process potentially changes the state of the environment and results in some reward for the learner. All that the learner needs to be provided with is the action space and a suitable reward function to determine an optimality metric.

about the environment are effectively a black-box. The agent takes an action, the environment then changes as a result according to some rule-set the learner need not have access to, and a reward is issued. The reinforcement learner then evolves to maximize total cumulative reward, not just the immediate reward benefit. Importantly, the environment may be governed by a deterministic set of differential equations, by a stochastic agent-based model, or by rules entirely determined by data. In this capacity, the black-box nature of the reinforcement learner environment is enticing to applied mathematicians as it allows the capacity to perform numerical learning experiments in a regime that was previously untractable. Indeed, recent advancements in computing power have allowed for the tractability of model-free reinforcement learning ${ }^{9}$. With the advent of big data sets and quantitative medicine, reinforcement learning can be used to leverage real world data as well as deterministic, validated models in order to learn a control in complicated contexts. Presently, we consider the environment to be governed by a system of simple differential equations to establish a framework methodology that can, in the future, be extended to other, more realistic domains.

In this study, we consider a simple differential equation model from Refs. ${ }^{1,10-12}$. This phenomenological model describes the growth of breast and ovarian solid tumours at a cellular level within a particular patient. The parameters of the model describe rates of cell-to-cell interaction and are incredibly difficult to measure in practice. In particular, the methods used in Refs. ${ }^{1,11}$ to parameterize this model only allow the discovery of nominal, mean values of such parameters from multiple mouse models. While these parameters can help to capture the qualitative behaviour of the response of a tumour to a particular chemotherapeutic, the model can certainly not be considered to be a validated model in human cancers. However, even for a validated phenomenological model the issue of patient-specific parameter identification still remains. Whenever the parameter values used for these models are determined by population-level data the modeller may not know $a$-priori the particular patientspecific parameters. In contexts where there is a demonstrable sensitivity to small perturbations in the parameter values, there is a concern that the nominal parameters (and any chemotherapeutic control thereby derived) may not robustly describe the most optimal response for a particular parameterization. To that end, in this paper we explore how chemotherapeutic controls derived from mean value parameters can be used on models of patients with perturbed, unknown parameter values. In particular, we leverage the power of deep double Q learning ${ }^{6}$ to derive the chemotherapeutic control in a manner that provides learned dosing schedules that are robust to perturbations in parameter values in this sensitive system. Importantly, the reinforcement learning agent is unaware during training of the patient-specific parameter values on which it is evaluated. This is in contrast to Ref. ${ }^{2}$ where multiple agents were trained on systems encoded by these parameter values exactly. In Ref. ${ }^{13}$ a continuous control problem is considered for both single and combination therapy of chemotherapeutics where the dynamics are described by an Ito stochastic differential equation (SDE). Importantly, the author employs a reinforcement learning method (the deep deterministic policy gradient method ${ }^{14}$ ) and notes that the corresponding control appears robust to the stochasticity inherent in the SDE. In Ref. ${ }^{1}$ the authors analytically derive the continuous optimal control of the tumour growth model used in this work under a particular objective functional. Here we consider a similar optimal control problem but wherein both the drug dose and time are discretized.

The manuscript is organized as follows. In the "Methods" section we first introduce the differential equation model and provide the mean parameter values that comprise the nominal virtual patient. We then define the optimal control problem considered and the objective functional by which the optimal scores are deduced. We then describe the method by which virtual patients were created for testing and training purposes. Next, we lay out the training process used for solving the reinforcement learning problem and the discrete optimal control problem. Finally, we discuss the hyperparameter tuning process of the deep double Q learning algorithm.

In the "Results" section, we first derive an analytic characterization of the initial conditions used in the model simulations as a function of these parameter values. Then, we perform local sensitivity analysis demonstrating that the model we consider is quite sensitive to local perturbations of the parameter values. We next present the results of the control agents by first allowing the agents to learn offline on an environment parameterized by the 


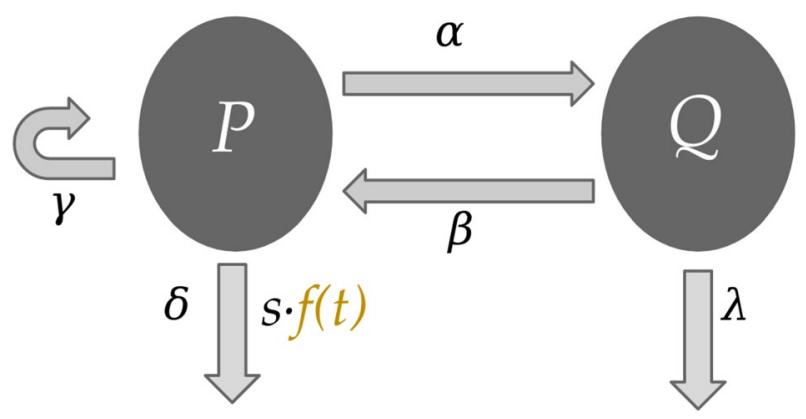

Figure 2. The two-compartment tumour growth inhibition model described by Eq. (1). Proliferative $(P)$ cells and quiescent $(Q)$ cells can both die naturally at the constant rates $\delta$ and $\lambda$, respectively. However, only proliferative cells can self-renew (at the constant rate $\gamma$ ) and be killed by the dose of a chemotherapeutic $f(t)$. Moreover, proliferative cells are allowed to become quiescent (at constant rate $\alpha$ ) and quiescent cells are allowed to become proliferative (at constant rate $\beta$ ).

nominal patient. We then apply these agents to environments parameterized by the perturbed testing patients. We measure the optimality of these schedules by logging the value of the objective functional achieved divided by the theoretical maximal value of this objective functional for each testing patient. We consider the optimality of schedules proposed for these perturbed testing patients for both the reinforcement learning agent and the traditional nominal optimal controller. In the former case, the relative bone marrow of these unknown patients was leveraged for the purpose of customizing the dosing schedule and reducing drug toxicity whereas, in the latter case, the same, nominal schedule is applied to all testing patients. We then extend the optimal schedules to leverage relative bone marrow measurements as well by employing a version of nearest neighbour interpolation on the optimal control of various training patients (whose particular patient specific parameter values are treated as known) to personalize dose schedules for testing patients. This nearest testing neighbour optimal control is then compared with the previous reinforcement learning agent. Finally, we present commentary and a summary of the work in the "Discussion" section.

\section{Methods}

Tumour growth inhibition model. We consider the two-compartment mathematical model of cell-cycle specific chemotherapy first introduced in Ref. ${ }^{11}$, which is an extension of earlier work ${ }^{10}$. The model consists of a population of proliferating cells and a population of quiescent cells, where the time evolution of cell populations is depicted in Fig. 2 and is governed by the following set of coupled ordinary differential equations:

$$
\begin{aligned}
& P^{\prime}(t)=(\gamma-\delta-\alpha-s f(t)) P(t)+\beta Q(t) \\
& Q^{\prime}(t)=\alpha P(t)-(\beta+\lambda) Q(t)
\end{aligned}
$$

In the model, $P(t)$ represents proliferative cells and $Q(t)$ represents quiescent cells. The model captures the growth of proliferative cells at a constant rate $\gamma$, the transformation of proliferative cells into quiescent cells at a constant rate $\alpha$, and the apoptosis of proliferative cells at a constant rate $\delta$. Similarly, quiescent cells leave quiescence and become proliferative at a constant rate $\beta$ and undergo apoptosis at a constant rate $\lambda$. The timedependent function $f(t)$ represents the dosing schedule of a chemotherapeutic where $s$ represents the relative strength of the administration of such a chemotherapeutic. In particular, it is assumed that $f(t) \in[0,1]$. While parameters $\gamma, \delta, \alpha, \beta$, and $\lambda$ are patient-specific parameters depending on the nature of the disease being modelled, parameter $s$ is a phenomenological hyper-parameter of the model describing the relative strength of the chemotherapeutic.

The proliferating cell compartment contains cells at each of the four phases of cell cycle (gap period G1, synthetic period S, second gap period G2, and mitosis M) to reduce the complexity of the cellular states. While resting cells are affected to a small extent by cell-cycle specific chemotherapy, the model(1) assumes that the chemotherapy $f(t)$ affects only the proliferating cells. The model does not include details from other aspects of the patient's context, notably it ignores the effects of age, sex, spatial information of the tumour, and any applicable comorbidities.

In Ref. ${ }^{10}$ the authors parameterize Eq. (1) with values that are suitable for describing breast cancer and ovarian cancer, as determined by mouse models. In Ref. ${ }^{1}$ the authors provide an additional parameter set for determining the effect of chemotherapy on healthy bone marrow cells. This allows one to, for a given chemotherapy dosing schedule $f(t)$, model the effect of chemotherapy on both the healthy bone marrow cells and the malignant cancerous cells. Hence, by evolving two de-coupled copies of Eq. (1), one parameterized with values corresponding to a particular cancer and the other with bone marrow parameter values, we can monitor the cancer-killing effects of a chemotherapeutic schedule and the associated chemotherapeutic toxicity in the patient. The parameter values are summarized in Table 1. 


\begin{tabular}{|c|c|c|}
\hline Parameter & Nominal value & Units \\
\hline \multicolumn{3}{|c|}{ Bone marrow } \\
\hline$\gamma$ & 1.470 & days $^{-1}$ \\
\hline$\delta$ & 0.000 & days $^{-1}$ \\
\hline$\alpha$ & 5.643 & days $^{-1}$ \\
\hline$\beta$ & 0.480 & days $^{-1}$ \\
\hline$\lambda$ & 0.164 & days $^{-1}$ \\
\hline$\rho_{p}^{*}$ & 0.103 & - \\
\hline \multicolumn{3}{|c|}{ Breast cancer } \\
\hline$\gamma$ & 0.500 & days $^{-1}$ \\
\hline$\delta$ & 0.477 & days $^{-1}$ \\
\hline$\alpha$ & 0.218 & days $^{-1}$ \\
\hline$\beta$ & 0.050 & days $^{-1}$ \\
\hline$\lambda$ & 0.000 & days $^{-1}$ \\
\hline$\rho_{p}^{*}$ & 0.200 & - \\
\hline \multicolumn{3}{|c|}{ Ovarian cancer } \\
\hline$\gamma$ & 0.6685 & days $^{-1}$ \\
\hline$\delta$ & 0.4597 & days $^{-1}$ \\
\hline$\alpha$ & 0.2225 & days $^{-1}$ \\
\hline$\beta$ & 0.0500 & days $^{-1}$ \\
\hline$\lambda$ & 0.0000 & days $^{-1}$ \\
\hline$\rho_{p}^{*}$ & 0.3600 & - \\
\hline
\end{tabular}

Table 1. Parameter values for breast cancer cells, ovarian cancer cells, and bone marrow cells as obtained from Refs. ${ }^{1,10}$, and values of $\rho_{p}^{*}$ as determined by Eq. (10).

Chemotherapeutic control. To determine the optimal chemotherapeutic control, we follow Ref. ${ }^{1}$ and introduce an objective functional with the form

$$
J_{b}(f)=\int_{0}^{T}\left[P_{\mathrm{bm}}(t)+Q_{\mathrm{bm}}(t)-\frac{b}{2}(1-f(t))^{2}\right] \mathrm{d} t .
$$

Maximizing this objective functional enables the derivation of an optimal chemotherapy dosing schedule of duration $T$ days for a particular patient. In the notation of Eq. (2), $P_{\mathrm{bm}}$ and $Q_{\mathrm{bm}}$ refer to the proliferative and quiescent compartments of Eq. (1) parameterized to describe the behaviour of bone marrow. In effect, this leads to a chemotherapeutic schedule that biases the optimizer toward applying a larger dose of chemotherapeutic, as governed by $f(t)$, while also maximizing the total number of bone marrow cells in the patient (to reduce drug toxicity). The non-negative hyperparameter $b$ is then a scaling factor representing the relative importance of these two mechanisms. If $b \gg 1$, then delivering the largest chemotherapeutic dose possible is the most desirable action for the optimizer, even at the cost of decimating the bone marrow cell count. Contrarily, if $0 \leq b \ll 1$ then the optimizer is biased toward preserving bone marrow even at the cost of lower cancer kill. Plots of such a chemotherapeutic dosing function $f$, obtained via the analytical method for deriving the continuous optimal control as in Ref. ${ }^{1}$, for various $b$ values are presented in Fig. 3.

In this work, the particular functional form of Eq. (2) is not of primary concern. Certainly other forms could be suggested to achieve similar qualitative goals. For instance, consider the functional

$$
J_{b}(f)=\int_{0}^{T}\left[P_{\mathrm{bm}}(t)+Q_{\mathrm{bm}}(t)-b\left(P_{\mathrm{bc}}(t)+Q_{\mathrm{bc}}(t)\right)\right] \mathrm{d} t .
$$

In the notation of Eq. (3), $P_{\mathrm{bc}}$ and $Q_{\mathrm{bc}}$ refer to the proliferative and quiescent compartments of Eq. (1) parameterized to describe the behaviour of solid breast cancer tumours. Hence, the functional in Eq. (3) describes the minimization of breast cancer cells while preserving the healthy, bone marrow cells. In this functional, the dependence on the chemotherapeutic dosing schedule $f$ is implicitly included in the trajectories of $P_{\mathrm{bm}}, Q_{\mathrm{bm}}$, $P_{\mathrm{bc}}$, and $Q_{\mathrm{bc}}$. In any case, the exact formulation of this objective functional is an incredibly important choice for any modeller in a clinical context as it determines the metric by which the control is considered maximal and is outside the scope of this report.

While there are many methods in the field of optimal control theory that provide a methodology for obtaining such schedules, one could also employ techniques from reinforcement learning to discover chemotherapeutic dosing schedules. For instance, for a time $t$ given the state vector $s_{t}$, to be defined later, and chemotherapeutic dose $a_{t} \in[0,1]$, we define the immediate reward function as 


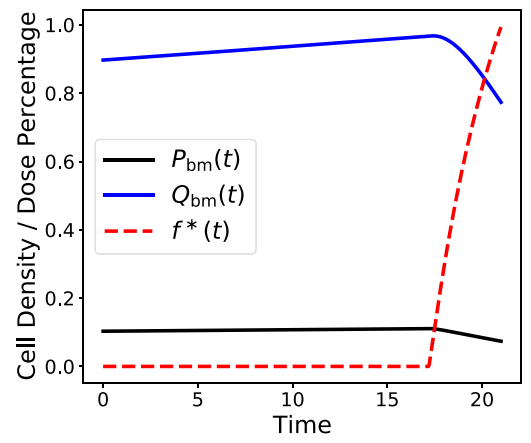

(a) $b=0.5$

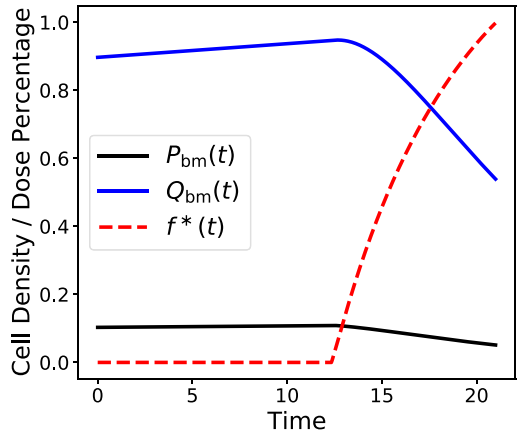

(b) $b=1$

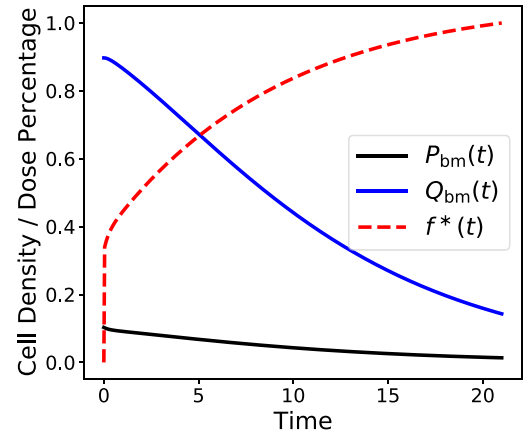

(c) $b=2$

Figure 3. A plot of the proliferative cell proportion (black), the quiescent cell proportion (blue), and the optimal chemotherapeutic control $f^{*}(t)$ (dashed red) for different values of $b$. The objective functional used to achieve this optimal control is given via Eq. (2). Small values of $b$ correspond to weighting preservation of the bone marrow as more important and larger values of $b$ correspond to weighting total drug delivery as more important.

$$
R\left(s_{t}, a_{t}\right)=\int_{t}^{t+1}\left[P_{\mathrm{bm}}(s)+Q_{\mathrm{bm}}(s)-\frac{b}{2}\left(1-a_{t}\right)^{2}\right] \mathrm{d} s
$$

in order to elicit an analogous response in the reinforcement learner as achieved by the objective functional in Eq. (2). Explicitly, $J_{b}(f)=\sum_{t=0}^{T-1} R\left(s_{t}, a_{t}\right)$, where $J_{b}$ is given in Eq. (2) for appropriate piecewise constant functions $f$. To proceed, we use the reward function in Eq. (4) in the following form of the Bellman equations (see, for instance, Ref. ${ }^{15}$ )

$$
Q^{*}\left(s_{t}, a_{t}\right)=R\left(s_{t}, a_{t}\right)+\gamma \sum_{s^{\prime} \in \mathbb{S}} p\left(s^{\prime} \mid s_{t}, a_{t}\right) Q^{*}\left(s^{\prime}, \underset{a^{\prime} \in \mathbb{A}}{\operatorname{argmax}}\left(Q^{*}\left(s^{\prime}, a^{\prime}\right)\right)\right)
$$

to derive an optimal policy as defined by

$$
\pi\left(s_{t}\right)=\underset{a \in \mathbb{A}}{\operatorname{argmax}} Q^{*}\left(s_{t}, a\right) .
$$

This policy can then be used to derive an optimal chemotherapy dosing schedule according to

$$
f^{*}(t)=\pi\left(s_{t}\right)
$$

We provide a brief explanation of Eqs. 5-7 but direct readers to a more thorough source such as Ref. ${ }^{15}$ for full details. As mentioned earlier, $R\left(s_{t}, a_{t}\right)$ represents the immediate reward an agent receives for performing action $a_{t}$ while in state $s_{t}$. In contrast, $Q^{*}\left(s_{t}, a_{t}\right)$ represents a valuation of performing action $a_{t}$ while in state $s_{t}$. Notably, $Q^{*}\left(s_{t}, a_{t}\right)$ encodes the immediate reward $R\left(s_{t}, a_{t}\right)$, but also encodes the discounted future rewards. Similarly, for a given $Q^{*}$ function the policy $\pi\left(s_{t}\right)$ describes the optimal action to perform in a given state $s_{t}$. As a result, $\pi\left(s_{t}\right)$ chooses an action $a_{t}$ to maximize $Q^{*}\left(s_{t}, a_{t}\right)$ in a global manner as compared to the local process of choosing $a_{t}$ to maximize immediate reward $R\left(s_{t}, a_{t}\right)$. As such, the maximal action in a given state, as valued by $Q^{*}$, may be one for which the payoff is not immediately obvious for multiple timesteps. The factor $\gamma$ in Eq. 5 is the discount factor of future rewards. The parameter $\gamma$ is taken such that $\gamma \in[0,1]$ where $\gamma=0$ corresponds to an agent that is focused on maximizing the immediate reward of their action and $\gamma=1$ corresponds to an agent more concerned with increasing future reward than immediate. In general, a model describing a reinforcement learning environment may not be deterministic. In that regard, $p\left(s^{\prime} \mid s_{t}, a_{t}\right)$ corresponds to the probability of ending up in state $s^{\prime}$ after taking action $a_{t}$ in state $s_{t}$. For the model in Eq. 1, no such stochasticity exists. As such, it is assumed that $p\left(s^{\prime} \mid s_{t}, a_{t}\right)=1$ for exactly one $s^{\prime} \in \mathbb{S}$ (namely $s^{\prime}=s_{t+1}$ ). One can derive the drug dosing schedule $f^{*}(t)$ as in Eq. 7 by observing the state in some manner and then evaluating the policy at this state. For the case of the nominal patient, where the patient specific parameters are known, observing the state is as simple as integrating Eq. 1. For an already trained model, one would construct the state vector (Eq. 8) for a patient (virtual or otherwise) and then evaluate the policy at this state.

For a continuous time reinforcement learning agent, the optimal dosing schedule learned by this process for a given parameterization of Eq. (1) is identical to that derived via optimal control theory for that same parameterization, as in Ref. ${ }^{1}$. Of particular importance, however, is that as Eq. (7) demonstrates, once a policy has been learned, one can derive an optimal chemotherapy schedule by merely evaluating the policy at the state. Importantly, the state one evaluates the policy at need not be a state seen during the learning process. Indeed, in our study we concern ourselves with training the reinforcement learning agent on only the nominal virtual patient and developing chemotherapy schedules for 200 different testing virtual patients. By leveraging state vector information from these 200 different testing virtual patients (patients which encode an environment over which the agent has not trained) the reinforcement learning agent is able to personalize the dose delivery function. As a result, it is important that we define our state vector as something that is both practically measurable 


\begin{tabular}{|c|c|c|}
\hline Parameter & Value & Description \\
\hline \multicolumn{3}{|c|}{ Hyperparameter Values } \\
\hline $\mathrm{hd}_{1}$ & 64 & Dimension of first hidden layer in the neural network \\
\hline $\mathrm{hd}_{2}$ & 96 & Dimension of second hidden layer in the neural network \\
\hline$\gamma$ & 0.9553 & Discount factor from the Bellman equation (5) \\
\hline$\alpha$ & 0.003809 & Learning rate for the Adam optimizer \\
\hline bs & 96 & Batch size for the Adam optimizer \\
\hline wl & 10 & Window length for relative bone marrow measurements \\
\hline
\end{tabular}

Table 2. Hyperparameter values for the learning process. The parameters $\mathrm{hd}_{1}, \mathrm{hd}_{2}, \gamma, \alpha$, and bs were determined by the Bayesian optimizer whereas wl was chosen empirically.

and phenomenologically linked to the objective functional we wish to optimize. As indicated by Eq. (8), when deriving the optimal chemotherapy dosing schedule according to Eq. (7), we are passing the optimal policy a w 1 length window of measurements corresponding to bone marrow count relative to a time before treatment began as well as the current day of treatment (in order to satisfy the Markov property).

$$
s_{t}=\left\langle t, P_{\mathrm{bm}}(t)+Q_{\mathrm{bm}}(t), P_{\mathrm{bm}}(t-1)+Q_{\mathrm{bm}}(t-1), \ldots, P_{\mathrm{bm}}(t-\mathrm{w} l+1)+Q_{\mathrm{bm}}(t-\mathrm{w} l+1)\right\rangle
$$

The particular value of $\mathrm{w} l$ is another hyperparameter to the process to consider. In contrast with the other hyperparameters listed in Table 2 , this hyperparameter was chosen empirically to be $\mathrm{w}=10$ as in Ref. ${ }^{2}$ wherein the authors used a length 10 window of mean tumour diameters as the state vector of their learning agent.

Perturbed virtual patients. We generate sets of virtual patients according to the following strategy. We consider parameter values from Table 1 and construct virtual patients by perturbing these parameter values. To begin we note that $\delta=0$ for the bone marrow parameters. As a result, we do not consider perturbing this value and treat $\delta$ as zero for all virtual patients. These perturbations are performed by scaling the mean parameter values in Table 1 by factors sampled from the space $[1-k, 1+k]$ uniformly with Latin hypercube sampling ${ }^{16}$. Latin hypercube sampling, a space filling technique for drawing random samples, is especially important when the number of samples drawn is small in comparison to the size of the sample space and when the parameters of interest are uncorrelated. Given the phenomenological nature of the remaining parameters we can assert that these parameters are uncorrelated. In particular, modifying any one of these parameters will create a distinct system under Eq. (1) that cannot be recovered by modifications to any number of the remaining parameters.

We now introduce some notation for describing the virtual patients. To begin, we let $\xi_{0}$ represent the nominal virtual patient. That is, $\xi_{0}=\left(\gamma_{\mathrm{bm}}, \delta_{\mathrm{bm}}, \alpha_{\mathrm{bm}}, \beta_{\mathrm{bm}}, \lambda_{\mathrm{bm}}\right)$ from Table 1 where the "bm" subscript refers to the bonemarrow parameter values. In this work, we generated virtual patients at perturbation levels of $k=0.15,0.20$, and 0.25 , where $k$ corresponds to the percent-change strength of perturbation. We generated six total sets of virtual patients. For the purpose of interpolating the nearest training neighbour optimal controller, we generated 1000 virtual patients at the $15 \%, 20 \%$, and $25 \%$ perturbation strength level for testing purposes which we denote by $\zeta_{i}^{k}$ where $1 \leq i \leq 1000$ denotes the index of the virtual patient and $k \in\{0.15,0.20,0.25\}$ denotes the maximal strength of the perturbation. Similarly, we generated 200 virtual patients at the $15 \%, 20 \%$, and $25 \%$ perturbation strength level for the purpose of testing the controllers, these patients we denote by $\theta_{i}^{k}$ where $1 \leq i \leq 200$ again represents the index of the virtual patient and $k \in\{0.15,0.20,0.25\}$ represents the maximal strength of the perturbation. The reinforcement learning agent was only trained on the nominal virtual patient and not virtual patients from the training or testing sets. The training virtual patients were only utilized for the crafting of the NTNOC optimal control strategy discussed in the "Results" section. In this regard, the testing virtual patients serve as a metaphor for the unknown patient-specific parameters of any particular patient in clinic. Both the testing and training virtual patients, for the non-zero parameters $\gamma, \alpha, \beta$, and $\lambda$, are visualized in Fig. S1.

Training process. We numerically solve the Bellman equation, Eq. (5), by employing neural networks as in the deep double Q-learning algorithm ${ }^{6}$. Deep double Q-learning is not the only algorithm by which one can solve this form of the Bellman equation. We chose this algorithm for a number of reasons: primarily, we expect that both the presence of the experience-replay buffer and the $Q(s, a)$ valuation is crucial for the algorithm to be able to successfully approximate the optimal action in the presence of environmental noise. Indeed, the success of this method relies on the ability of the algorithm to approximate the value of state/action pairs that differ from those seen in the optimal treatment of the nominal patient. By maintaining a buffer and valuation of the state/ action pairs explored while deriving the nominal treatment, the algorithm is able to better approximate a larger swathe of the domain of $Q$. There are many algorithms that satisfy these requirements such as deep deterministic policy gradient or (single) deep Q-learning ${ }^{14,17}$. However, deep policy gradient is a more computationally expensive method that can produce continuous controls, whereas we are primarily concerned with discrete dose values in this study. Similarly, deep Q-learning has been observed to be more likely to select overestimated values, resulting in overtly optimistic value estimates, in a capacity that is avoided in deep double Q-learning ${ }^{6}$. In particular, we represent the $Q$ function from the Bellman equation, Eq. (5), as a neural network. As a result, after training the network, the specific form of $Q$ is the same for each testing virtual patient. However, as in Eq. (7), by supplying the bone marrow measurements for the testing virtual patient, the network can produce a person- 


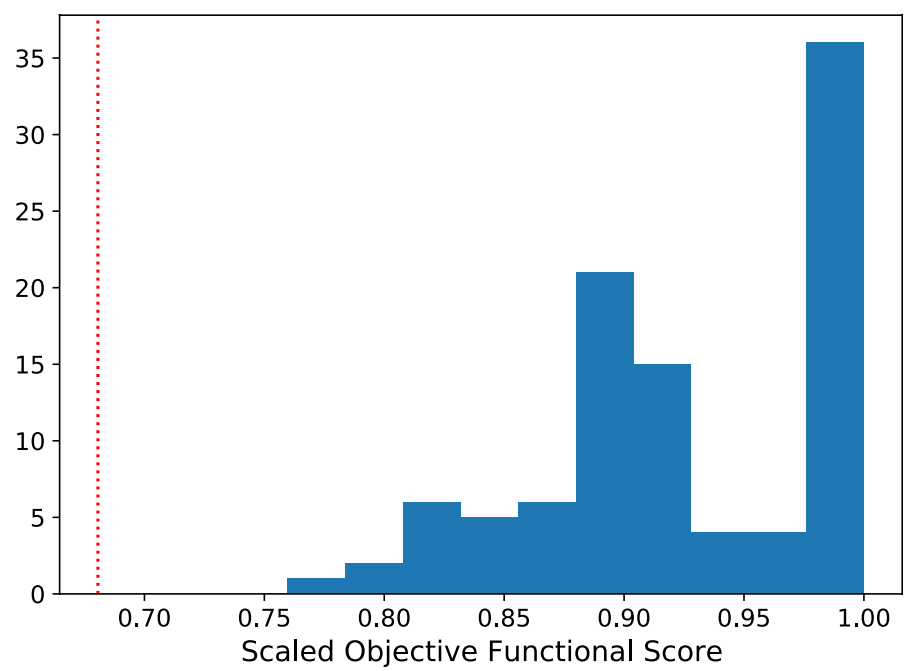

Figure 4. A histogram demonstrating all the scores obtained via the reinforcement learning process. The red dotted line indicates the expected score of a random agent.

alized dose schedule that is different for each virtual patient. In terms of the architecture of the $Q$ network, we take the network to have 10 inputs neurons (as determined by the length of the state vector), $\mathrm{hd}_{1}$ neurons in the first hidden layer, $\mathrm{hd}_{2}$ neurons in the second hidden layer, and 11 neurons in the output layers (corresponding to dose strength range from 0 to 1 inclusive in 0.1 increments). Each neural layer is activated with a rectified linear unit. We use batch-learning with a batch size of bs to minimize the mean squared error between the right and left hand sides of Eq. 5 via an Adam optimizer with learning rate $\alpha$. In the Hyperparameter Tuning section we discuss how we decide upon the values of these hyperparameters and list the particular values in Table 2 . To train the network we first parameterize the system in Eq. 1 by the nominal parameter set $\xi_{0}$. Next, we run 5,000 exploratory time steps of the simulation performing random actions in order to fill the experience replay buffer. After every 21 time steps, the environment is reset by returning the differential equation model, Eq. (1), back to its initial conditions (as dictated by Eq. (10)). In particular, the initial state for the reinforcement learning algorithm is a length eleven vector where the first entry is a 0 and the remainder are unit entries (as the Eq. (10) initial conditions sum to 1 ). This window length of 10 for the bone marrow measurements was chosen empirically, as in Ref. ${ }^{2}$. For each time step of the simulation we choose a chemotherapy dose according to our network via an $\epsilon$-greedy algorithm. We anneal $\epsilon$ linearly from $\epsilon=1$ to $\epsilon=0.01$ over 25,000 time steps. The $\epsilon$-greedy algorithm was only implemented during training, i.e. during evaluation the policy selection is deterministic as in Eq. (6). After selecting a dose $a \in \mathbb{A}=\{0,0.1, \ldots, 1\}$, we apply the chemotherapy dose to the patient by holding $f(t)=a$ constant over the timestep and evolving Eq. (1) (as such, we discretize not only in dose but in time as well). Next, we record a tuple of the state, action, reward, new state values. We then select a random batch of previously observed tuples and use them to approximate the right hand side of the Bellman equation, Eq. (5), in order to obtain a target for training the network.

This process is eventually stopped if the differential equation environment has been reset 50,000 times or if the best reward has not improved over the last 500 epochs. This constitutes one training run of the system. We perform 5 such training runs recording the run that achieved the largest objective functional score under Eq. 2.

Hyperparameter tuning. While our system has many model specific parameters, there are also a number of hyperparameters introduced during the training process. These are the learning rate for the Adam optimizer $(\alpha)$, the dimension of the two hidden layers ( $\mathrm{hd}_{1}$ and $\mathrm{hd}_{2}$, respectively), the discount rate $\gamma$ in the Bellman equation (Eq. (5)), and the batch size of the Adam optimizer used for learning (bs). In order to ensure optimal convergence and stability of the resultant networks, we must carefully select these values. For a single set of these five hyper-parameters we must execute the entire training process over again. Such a process is computationally extensive rendering a brute-force grid-search approach to hyperparameter optimization unfeasible. To that end, we instead use Bayesian optimization to explore this five-dimensional hyperparameter space more efficiently. We allow our Bayesian optimizer to sample 100 such hyperparameter samples from this hyperparameter space and perform the training process for each hyperparameter set. The Bayesian optimizer chose hd $\mathrm{and} \mathrm{h}_{2}$ from the set $\{64,96, \ldots, 256\}$, bs from the set $\{32,64, \ldots, 128\}, \alpha$ from the interval $\left(10^{-4}, 10^{-1}\right)$, and $\gamma$ from the interval $(0,1)$.

In Fig. 4 we see the distribution of the objective functional score under Eq. 2 for the 100 reinforcement learning agents under this hyperparameter tuning process. In particular, we note the cluster of 36 agents that converged to the network architecture with the theoretical maximal objective functional value, as determined by running a discretised version of the optimal control problem from Ref. ${ }^{1}$ with the APOPT algorithm (as implemented by $\mathrm{GEKKO}^{5,18}$ ). For a point of comparison, we calculated the expected score achievable by a random agent by calculating the mean value of the score obtained in 1,000,000 simulations where a dose from 

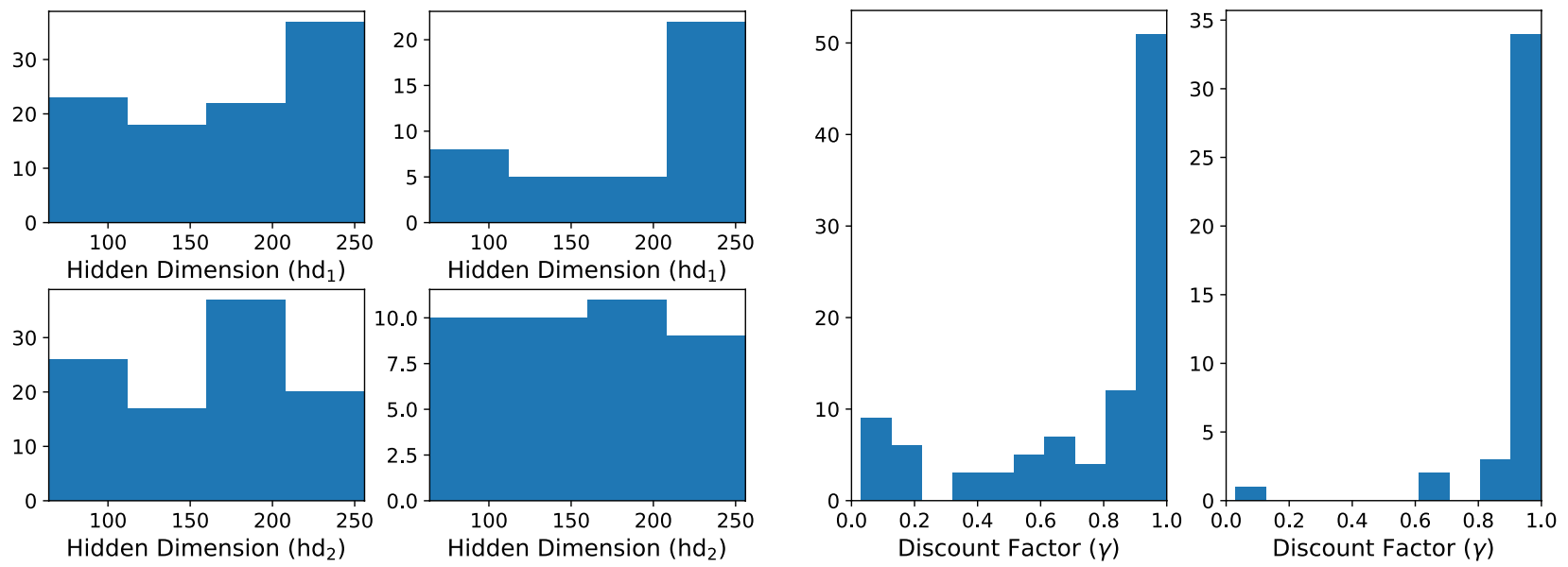

(a) Distributions of the size of the hidden dimensions of the neural network architecture.
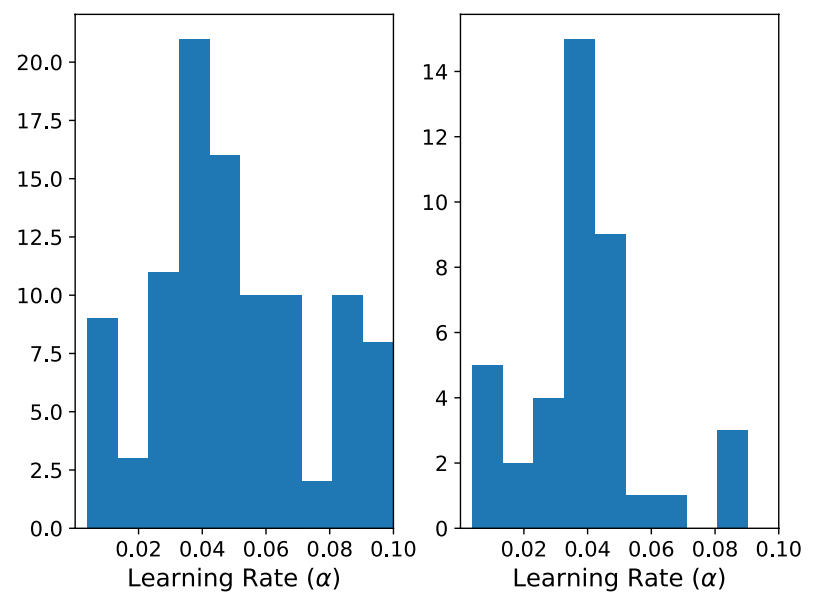

(b) Distributions of the discount factor $\gamma$ used in the Bellman equation, Eqn.(5).
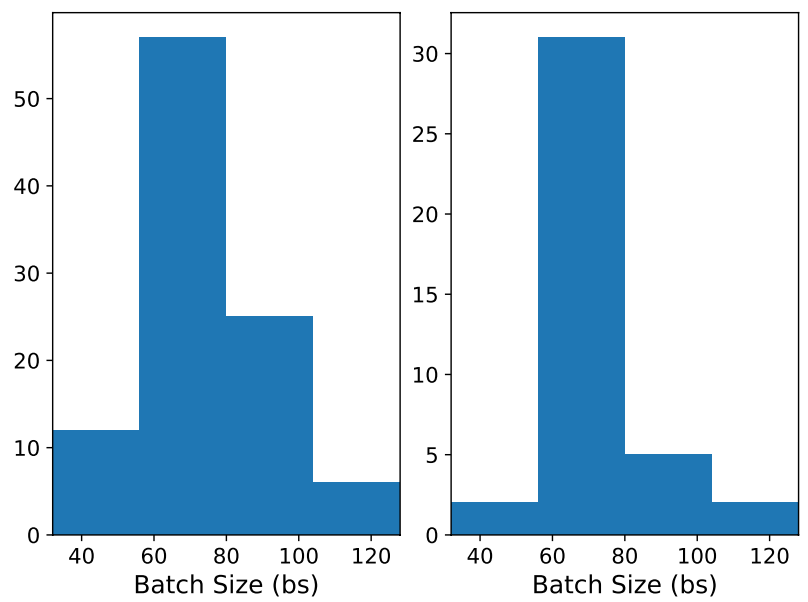

(c) Distributions of the learning rate used in theAdam optimizer for the neural network.

(d) Distributions of the batch size used in Adam optimizer for the training of the neural network.

Figure 5. In all figures, the distributions on the left represent the total distribution of the hyperparameter explored by the Bayesian hyperparameter optimizer. In contrast, the distributions on the right in each figure indicate the distribution of the hyperparameter conditioned on the objective functional value being within $5 \%$ of the maximal theoretical score.

$\{0,0.1, \ldots, 1.0\}$ was uniformly selected at each time step. This resulted in a mean objective functional value of 0.6806 with a standard deviation of the mean of 0.04811 .

To ascertain the identifiability and stability of these parameters, we consider the distribution of parameters that result in such objective functional value. To that end, in Fig. 5 we consider the distribution of each individual hyperparameter and contrast this with the distribution of such hyperparameters from the agents that converged to network architecture that achieve an objective functional value value within $5 \%$ of the maximal possible reward. Importantly, we recognize that of the five hyperparameters, there is not a tight distribution after conditioning on objective functional value score. In fact, only the discount factor $\gamma$ produces a conditioned distribution that is statistically different than the un-conditioned distribution (two-sample Kolmogorov-Smirnov $p$-value of approximately $0.001^{19}$ ). This suggests that the particular values of the size of the hidden dimensions, learning rate, and batch size are not terribly sensitive parameters for the training of this reinforcement learning agent.

The Bayesian optimizer determined an optimal hyperparameter choice of $\mathrm{hd}_{1}=64 \mathrm{hd}_{2}=96, \gamma=0.9553$, $\alpha=0.003809$, and bs $=96$. Though, as the above discussion demonstrates, it is only the choice of $\gamma$ that appeared to have any particularly strong impact on the convergence of the training process. A $\gamma$ value close to 1 can be interpreted as representing an agent with a far horizon ${ }^{15}$. In particular, such an agent is less concerned with the immediate reward of a particular action and more concerned with the long-term, cumulative reward obtained by maximizing Eq. 2 over all time. 


\section{Results}

Derivation of the proliferative fraction. We begin modelling the proliferative and quiescent components of Eq. 1 under the assumption that the tumour has evolved in the absence of any chemotherapeutic agent until a steady state, in terms of the proportion of these cells, has been reached. To that end, we define the proliferative ratio of the tumour at time $t$ and the steady-state proliferative ratio as

$$
\rho_{p}(t)=\frac{P(t)}{P(t)+Q(t)} \quad \text { and } \quad \rho_{p}^{*}=\lim _{t \rightarrow \infty} \rho_{p}(t),
$$

respectively. To analytically calculate the closed form solution of the steady-state proliferative ratio in the absence of a chemotherapeutic, we set $s=0$ and consider

$$
\begin{aligned}
0 & =\rho_{p}^{\prime}(t) \\
& =\frac{P^{\prime}(t)}{P(t)+Q(t)}\left(1-\rho_{p}^{*}\right)-\frac{Q^{\prime}(t)}{P(t)+Q(t)} \rho_{p}^{*} \\
& =(\delta-\gamma-\lambda) \rho_{p}^{* 2}+(\gamma+\lambda-\beta-\alpha-\delta) \rho_{p}^{*}+\beta .
\end{aligned}
$$

Thus, $\rho_{p}^{*}$ is a root of the quadratic in Eq. (9). For the parameter values presented in Table 1 the quadratic in Eq. (9) has only one positive (real) root, namely

$$
\rho_{p}^{*}=\frac{1}{2} \frac{-\lambda-\gamma+\alpha+\delta+\beta-\sqrt{(\lambda+\gamma-\alpha-\delta-\beta)^{2}-4(\delta-\lambda-\gamma) \beta}}{\delta-\lambda-\gamma} .
$$

The values of $\rho_{p}^{*}$ corresponding to the parameters for ovarian cancer, bone marrow, and breast cancer are included in Table 1 . Thus the initial data for Eq. (1) considered in this study are given by $P(0)=\rho_{p}^{*}$ and $Q(0)=1-\rho_{p}^{*}$.

Local sensitivity analysis. Here we investigate the sensitivity of the outputs for the tumour growth inhibition model, Eq. (1), to perturbations in the nominal parameter values of the model-specific parameters presented in Table 1 . To compute the sensitivities, we change the values of the parameters $\gamma, \delta, \alpha, \beta$, and $\lambda$ one-at-a-time by a small amount, $\Delta p$. We take $\Delta p$ to be $+1 \%$ of the nominal parameter value $p_{0}$. Then the relative sensitivity of each model population $x=\langle P(T), Q(T)\rangle$ for the parameter is calculated as follows:

$$
R_{x, p}=\frac{\left(x-x_{0}\right) / x_{0}}{(\Delta p) / p_{0}}
$$

where subscripts denote nominal values. The initial conditions of the simulations were recalculated according to Eq. (10) for each perturbed parameter value and the simulations were run until $T=21$ days. We plot the results in Fig. 6.

Importantly, the results of Fig. 6 indicate that the model exhibits substantial sensitivity due to relatively small perturbations in the patient-specific parameter values. This type of sensitivity is common in models that experience regimes of exponential growth, which are common in cellular models of cancer ${ }^{12}$. For the parameter sets corresponding to breast cancer, Fig. 6a indicates a mean (absolute) change of roughly $2.3 \%$ in $P$ cells, $0.97 \%$ in $Q$ cells, or $1.14 \%$ in all cell types given a $1 \%$ perturbation to a singular parameter. For ovarian cancer the model is even more sensitive, demonstrating a mean (absolute) change of roughly $5.3 \%$ in $P$ cells, $3.4 \%$ in $Q$ cells, or $4.1 \%$ in all cell types given a $1 \%$ perturbation to a singular parameter. For bone marrow, similar extreme sensitivities are observed with a mean (absolute) change of roughly $4.1 \%$ in $P$ cells, $3.5 \%$ in $Q$ cells, or $3.6 \%$ across all cell types. Importantly, Fig. 6 demonstrates that even for the least sensitive parameter set (the breast cancer parameter set), small perturbations to individual parameters can still elicit large differences in the evolution of a tumour if one is unlucky enough that such a perturbation occurred in either $\gamma$ or $\delta$ (the self-renewal and death rate of proliferative cells, respectively).

Contrasting a nominal reinforcement learning agent with a nominal optimal controller. We first begin by training a reinforcement learner on the nominal parameter set from Table 1 using the hyperparameters for the training method from Table 2. During training, the reinforcement learning agent only interacted with the environment from Eq. (1) parameterized by the nominal set. Similarly, as a point of comparison, we used the APOPT algorithm from the GEKKO Python library to solve the discretized optimal control problem on the nominal parameter set ${ }^{5,18}$. These two agents, one a reinforcement learning agent and the other a traditional optimal controller, were kept blind to the testing and training virtual patients. That is, the reinforcement learning agent was trained offline on an environment parameterized by $\xi_{0}$ before the policy derived was tested on environments parameterized by $\theta_{i}^{k}$ for all $i$ and $k$. Similarly, the traditional optimal control was derived for patient $\xi_{0}$ before being applied to the testing patients $\theta_{i}^{k}$ for all $i$ and $k$. We then applied chemotherapeutic dosing schedules derived from both methods on the 600 testing virtual patients (200 virtual patients each at the $15 \%, 20 \%$, and $25 \%$ perturbation strength level). We define $\sigma_{\mathrm{RL}}\left(\theta_{i}^{k} ; \xi_{0}\right)$ to refer to the value under the objective functional in Eq. 2 achieved by this reinforcement learning agent when applied to patient $\theta_{i}^{k}$ after training the agent on an environment parameterized by the nominal patient $\xi_{0}$. We similarly define $\sigma_{\mathrm{OC}}\left(\theta_{i}^{k} ; \xi_{0}\right)$ to be the score achieved by applying the optimal control for patient $\xi_{0}$ to patient $\theta_{i}^{k}$. In order to scale the scores of these trials, we separately solved the discretized optimal control problem on these testing virtual patients using the APOPT algo- 


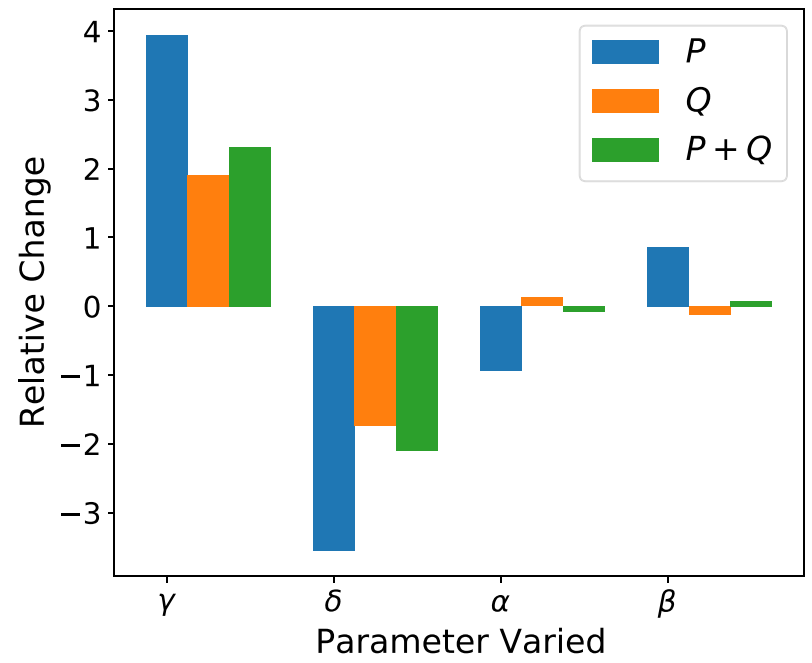

(a) Relative sensitivity of the breast cancer parameter values.

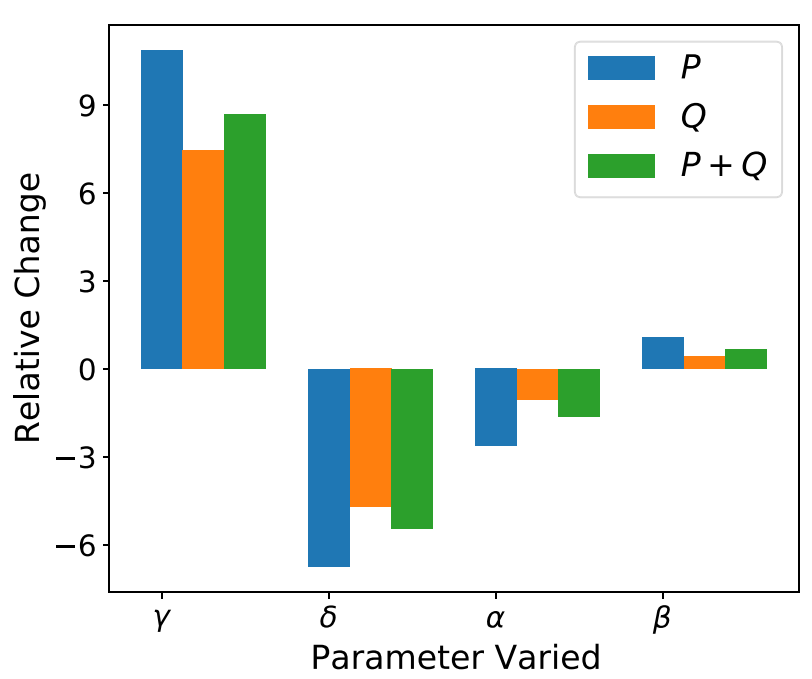

(b) Relative sensitivity of the ovarian cancer parameter values.

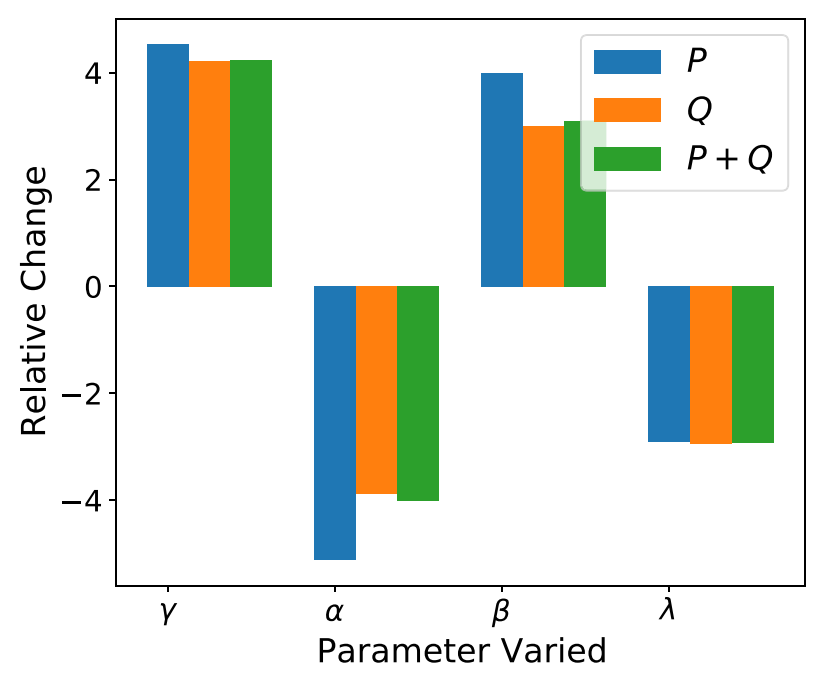

(c) Relative sensitivity of the bone marrow parameter values

Figure 6. Relative sensitivity of Eq. (1) under the parameter sets from Table 1. Parameters with zero value ( $\delta$ for bone marrow and $\lambda$ for breast and ovarian cancer) were ignored and not displayed in this figure.

rithm from GEKKO. Importantly, these 600 solutions were only used to ascertain the maximal possible objective functional value in order to scale the result of the blind agents. We define $\sigma\left(\theta_{j}^{k}\right)$ denote the maximal value of the objective functional in Eq. 3 when parameterized by patient $\theta_{i}^{k}$. We let $\hat{\sigma}_{\mathrm{RL}}\left(\theta_{i}^{k} ; \xi_{0}\right)=\sigma_{\mathrm{RL}}\left(\theta_{i}^{k} ; \xi_{0}\right) / \sigma\left(\theta_{i}^{k}\right)$ denote this scaled objective functional score. As a result, a score of 1 is the maximal score theoretically obtainable under the objective functional for the discrete problem by either solution method. In general, $\hat{\sigma}_{\mathrm{RL}}\left(\theta_{i}^{k} ; \xi_{0}\right) \leq 1$. Similarly $\hat{\sigma}_{\mathrm{OC}}\left(\theta_{i}^{k} ; \xi_{0}\right)=\sigma_{\mathrm{OC}}\left(\theta_{i}^{k} ; \xi_{0}\right) / \sigma\left(\theta_{i}^{k}\right) \leq 1$ represents the scaled score of the optimal control agent. Finally, we compared the blind agents results by applying their derived chemotherapy strategies to the testing virtual patients, scaling the output according to the previously ascertained maximal possible reward. The results of this are presented in Fig. 7 for the 3 different perturbation strength levels.

Notably, the chemotherapy dosing schedule determined via optimal control for the nominal parameter set is a particular function $f^{*}$ that is the same for each virtual patient. In effect, each virtual patient is treated with the therapy schedule that is optimal for the mean-valued patient. In contrast, in the reinforcement learning derived schedule, the policy from Eq. (6) is the same for each virtual patient, but that policy is being fed a 10 day window of relative bone marrow cell counts from each virtual patient as a state vector. In particular, the nominal optimal controller is an open-loop controller whereas the reinforcement learning agent is a feedback controller. As a result, we are allowing the reinforcement learner to refine its dosing schedule given this information. By doing so we are able to acquire a dosing schedule that is more robust to perturbations in these unknown, assumed to be unmeasurable, patient-specific model parameters by allowing refinements to be made based on a more easily measurable aggregate metric. Hence, the fact that the reinforcement learning agent is a feedback controller is exactly why it is able to utilize information from the state vector in the design of these dose delivery schedules. Importantly, the state vector for the reinforcement learner is the sum of the $P_{\mathrm{bm}}$ and $Q_{\mathrm{bm}}$ compartments of Eq. (1) 


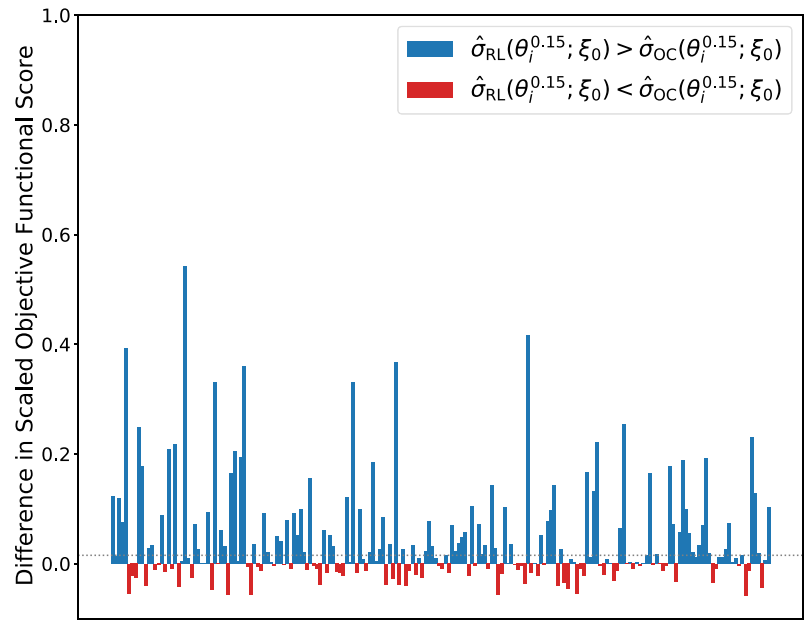

(a) Difference in scaled objective functional score for virtual patients at $15 \%$ perturbation strength.

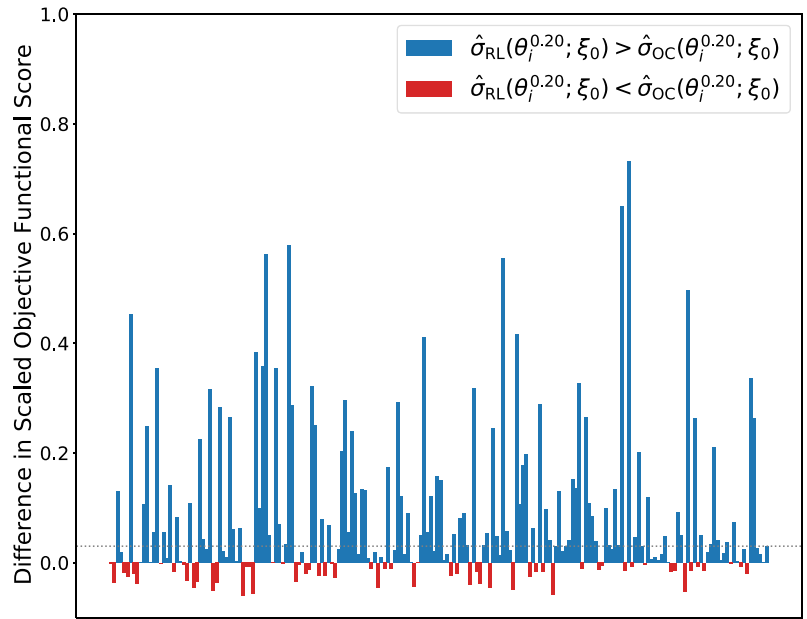

(b) Difference in scaled objective functional score for virtual patients at $20 \%$ perturbation strength.

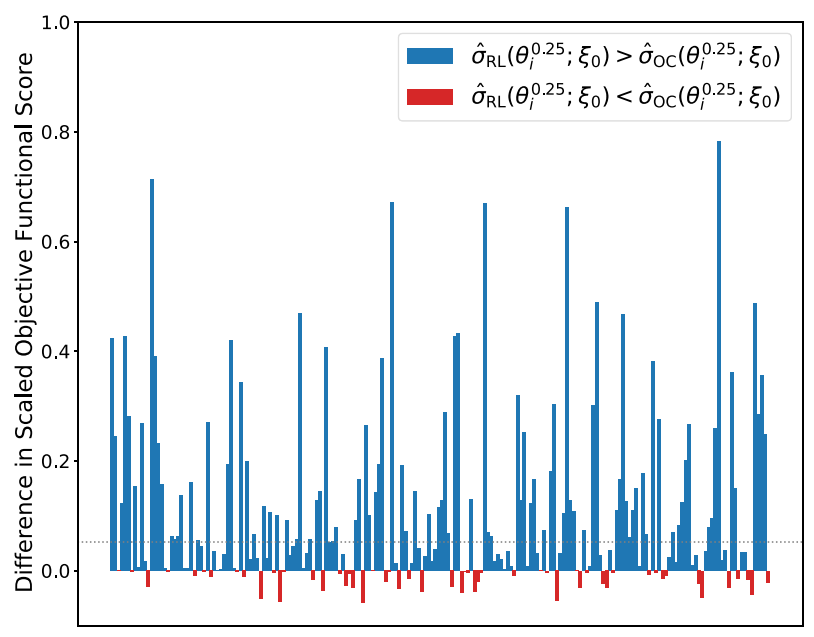

(c) Difference in scaled objective functional score for virtual patients at $25 \%$ perturbation strength.

Figure 7. Bar plots of the difference between the scores obtained by the reinforcement learner derived policy and the scores obtained by the optimal control derived policy on all test virtual patients (i.e. bar plots of $\left.\hat{\sigma}_{\mathrm{RL}}\left(\theta_{i}^{k} ; \xi_{0}\right)-\hat{\sigma}_{\mathrm{OC}}\left(\theta_{i}^{k} ; \xi_{0}\right)\right)$. Testing patients where the reinforcement learner outperformed the optimal controller are marked in blue and patients where the optimal controller outperformed the reinforcement learner are marked in red. The dotted grey lines in each plot indicate the difference of the median scaled scores of the reinforcement learner and the optimal controller.

at discrete time points (in this case, daily) and not the individual measurements of $P_{\mathrm{bm}}$ and $Q_{\mathrm{bm}}$ separately. Given the scaling of Eq. (1) under the initial conditions from Eq. (10), these measurements are taken relative to the bone marrow mass prior to treatment, and so absolute measurements are not required. See Fig. S4 for a visualization of these schedules on different virtual patients.

To quantify the performance differences of the two dose schedule processes over the testing virtual patients, we compared the distributions of scores with the non-parametric one-sided Wilcoxon signed-rank test ${ }^{20}$. For the cases represented in Fig. $7 \mathrm{a}-\mathrm{c}$ we considered the alternative hypothesis to be that the median scaled score obtained by the reinforcement learner is larger than the median scaled score obtained by the optimal controller (i.e. the alternative hypothesis is median $\left.\left(\hat{\sigma}_{\mathrm{RL}}\left(\theta ; \xi_{0}\right)\right)>\operatorname{median}\left(\hat{\sigma}_{\mathrm{OC}}\left(\theta ; \xi_{0}\right)\right)\right)$. We found at the $15 \%$ perturbation strength level a Wilcoxon statistic of 14681 corresponding to a $p$-value on the order of $10^{-9}$, at the $20 \%$ perturbation strength level we found a Wilcoxon statistic of 16,528 corresponding to a $p$-value on the order of $10^{-15}$, and at the $25 \%$ perturbation strength level we found a Wilcoxon statistic of 17,551 corresponding to a $p$-value below machine precision. In all cases, we reject the null hypothesis and conclude that the reinforcement learning agent produces chemotherapeutic schedules with a higher median score on perturbed patients than the optimal controller. We notice that as the perturbation strength increases, the difference in the median and mean scaled scores increases as well from a difference in medians of 0.011 in the $15 \%$ case (difference of means of 0.045 ) to 


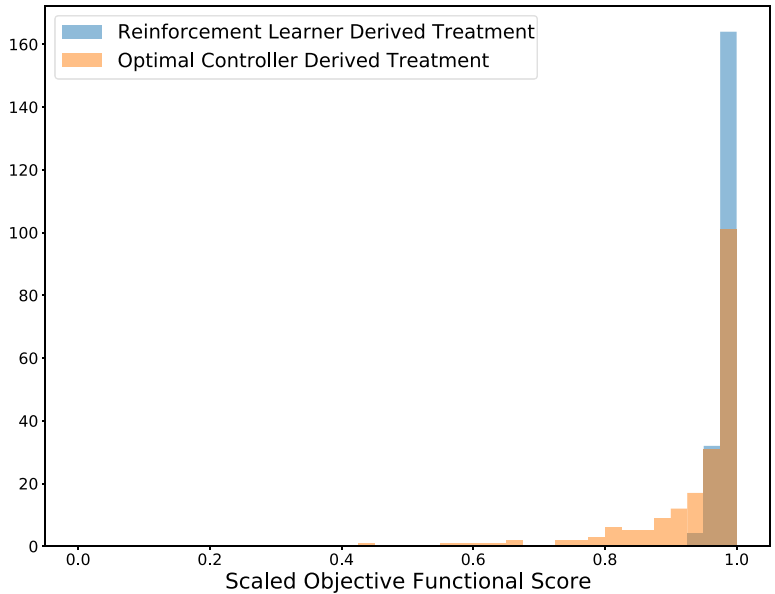

(a) At $15 \%$ perturbation strength the reinforcement agent produces a schedule that produces median scaled scores of 0.991 while the optimal controller derived schedule produces median scaled scores of 0.976 .

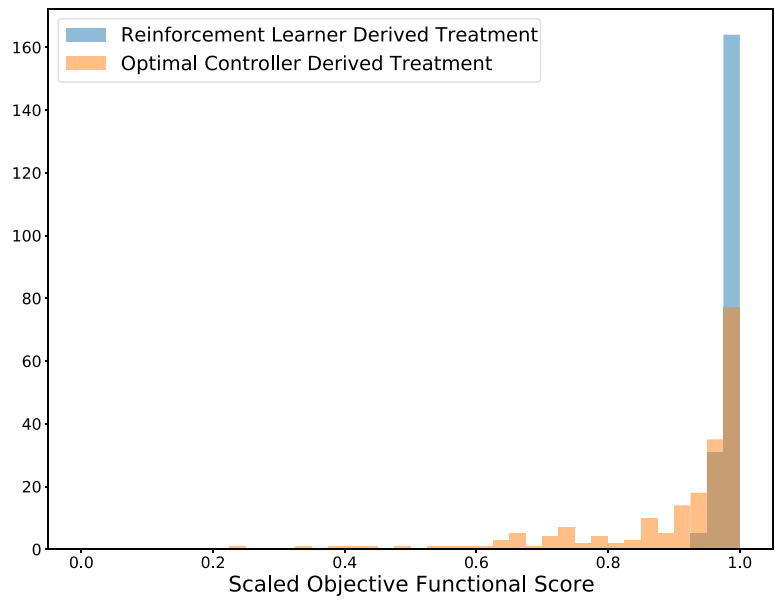

(b) At $20 \%$ perturbation strength the reinforcement agent produces a schedule that produces median scaled scores of 0.992 while the optimal controller derived schedule produces median scaled scores of 0.962 .

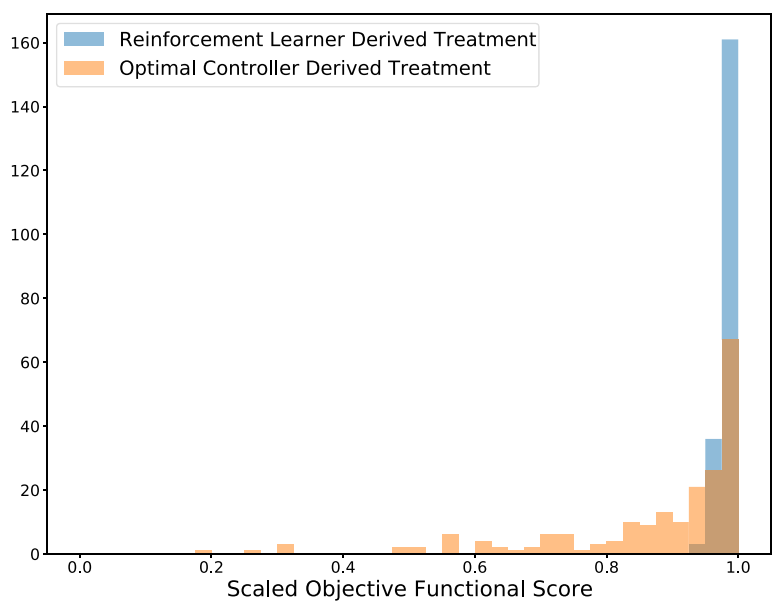

(c) At $25 \%$ perturbation strength the reinforcement agent produces a schedule that produces median scaled scores of 0.991 while the optimal controller derived schedule produces median scaled scores of 0.940 .

Figure 8. Histograms of the scores achieved by the various agents on the 200 testing virtual patients. Bin sizes were chosen to correspond to 0.025 . In particular, the reinforcement learning agent is much more robust toward perturbation in parameter values, consistently producing dosing schedules scoring within $7.5 \%$ of the theoretical maximal score.

a difference in medians of 0.044 (difference of means of 0.108 ) in the $25 \%$ case. Hence, as the strength of the perturbation increases over this range, the reinforcement learner outperforms the optimal controller even further.

In Fig. 8 we present the histograms of the scaled scores for each blind agent on the 600 different virtual patients. The histograms are semi-transparent in order to aid comparison where the blue colour represents the reinforcement learning agent and the orange colour the APOPT derived optimal controller agent. We note that the reinforcement learning agent has a large cluster of treatments in the $97.5-100 \%$ optimal bin (164 out of 200 in the $15 \%$ case, 165 out of 200 in the $20 \%$ case, and 161 out of 200 in the $25 \%$ case) and all treatments fall within $7.5 \%$ of the theoretical maximum. These scores are achieved without training on these virtual patients directly. In contrast, the optimal controller derived treatment is much more diffuse. As the strength of perturbation increases, the average score of the reinforcement agent derived schedule remains within $1.2 \%$ of optimum, while the average optimal control derived score decreases dramatically from 0.941 in the $15 \%$ case, to 0.902 in the $20 \%$ case, and finally to 0.879 in the $25 \%$ case. In particular, this suggests that the increase in performance of the reinforcement learner as a result of perturbation strength is due to the reinforcement learning agents ${ }^{\text {Tw }}$ capacity to remain non-sensitive to these perturbations, in contrast to the sensitivity seen in the schedules derived by the optimal controlling agent. 
Contrasting a nominal reinforcement learning agent with a nearest neighbour interpolated optimal controller. The results of the previous subsection indicate that the reinforcement learning agent produces schedules that are more robust to perturbations in the unknown parameters. We noted that the reinforcement learning agent is capable of customizing these schedules for each individual patient, not by measuring the patient specific parameters directly, but by customizing the response via a more easily measurable metric. In this section, we consider a different training process that allows the optimal controller agent a comparable level of customization.

For this comparison, we kept the reinforcement learning agent exactly the same as in the previous section: the agent was trained offline on an environment parameterized by $\xi_{0}$ before being applied as a test to environments parameterized by $\theta_{i}^{k}$ for all $k$ and $i$. For the optimal controller comparison, we begin by solving the discrete optimal control problem on all 1000 training virtual patients for each perturbation strength (i.e. the optimal control was determined for patient $\zeta_{i}^{k}$ for all $k$ and all $i$ ). We then log the state vector from Eq. (8) for each timestep of treatment. For a fixed perturbation strength $k$ we first calculated the state vector $s_{t_{i}}$ for each of the 200 testing patients. We then applied a chemotherapeutic dose by consulting the table of states from the training patients at time $t_{i}$. The dose was selected from the training patient whose state vector was closest to the current testing patient state vector (where distance was measured by the Euclidean metric). Note that the state vector is as in Eq. 8 and as such contains a length $\mathrm{w} l=10$ moving window of relative bone marrow measurements. The result of this nearest training neighbour optimal controller (NTNOC) was an agent that could also customize chemotherapeutic dosing strategies for each of the 200 testing virtual patients $\left(\theta_{i}^{k}\right)$ based off of knowledge gained by traversing the training virtual patient space $\left(\zeta_{i}^{k}\right)$. Hence, while the optimal controller presented in the previous section was an open-loop controller, the NTNOC is able to incorporate feedback from the environment. We define $\sigma_{\text {NTNOC }}\left(\theta_{i}^{k} ; \zeta^{k}\right)$ to represent the score obtained in an environment parameterized by patient $\theta_{i}^{k}$ under the objective functional in Eq. 2 achieved by an NTNOC agent trained on the set $\zeta^{k}=\left\{\zeta_{i}^{k} \mid 1 \leq i \leq 1000\right\}$. Similarly, we define $\hat{\sigma}_{\mathrm{NTNOC}}\left(\theta_{i}^{k} ; \zeta^{k}\right)=\sigma_{\mathrm{NTNOC}}\left(\theta_{i}^{k} ; \zeta^{k}\right) / \sigma\left(\theta_{i}^{k}\right) \leq 1$ to be the scaled objective functional score. The reinforcement learning agent that the NTNOC agent is being compared to only ever interacted with a differential equation environment parameterized by the nominal parameter set $\xi_{0}$. Ostensibly, more distribution level information is directly afforded to the NTNOC than was afforded to the reinforcement learning agent. The reinforcement learning agent is only able to customize treatment strategies based off the states learned by providing non-optimal doses to the nominal virtual patient $\left(\xi_{0}\right)$ during training. The results of this comparison are presented in Fig. 9. In particular, we note that the same general trend from Fig. 7 is repeated: namely, as the perturbation strength increases the relative performance of the reinforcement learning agent also increases. However, in contrast to Fig. 7, we note that at the $15 \%$ level the nearest training neighbour optimal controller outperforms the reinforcement learning agent (with a one-sided Wilcoxon signed-rank test p-value on the order of $10^{-5}$ ). Indeed, the mean value of the differences plotted in Fig. 9a occurs at approximately -0.007 , indicating that, in a mean value sense, the nearest training neighbour optimal controller produces strategies that are 0.007 points closer to the optimal score of 1 than the scores of the schedules produced by the reinforcement learning agent.

Again we compare the distributions of scores with the one-sided Wilcoxon signed-rank test, though for the case represented in Fig. 9a, we consider the alternative hypothesis to be that the nearest training neighbour optimal controller produces schedules with higher median scaled score than that of the reinforcement learning agent (i.e. the alternative hypothesis is median $\left(\hat{\sigma}_{\mathrm{NTNOC}}\left(\theta ; \zeta^{k}\right)\right)>\operatorname{median}\left(\hat{\sigma}_{\mathrm{RL}}\left(\theta ; \xi_{0}\right)\right)$ ). We found at the $15 \%$ perturbation strength level a Wilcoxon statistic of 6315 corresponding to a $p$-value on the order of $10^{-5}$. Hence we reject the null hypothesis and conclude that, at the $15 \%$ perturbation level, that the NTNOC produces chemotherapeutic schedules with higher median scaled score than those produced by the reinforcement learning agent. For the cases represented in Fig. 9b, $\mathrm{c}$ we consider a different alternative hypothesis: namely that the reinforcement learning agent produces chemotherapeutic schedules with higher median scaled score than those produced by the NTNOC (i.e. the alternative hypothesis is median $\left.\left(\hat{\sigma}_{\mathrm{RL}}\left(\theta ; \xi_{0}\right)\right)>\operatorname{median}\left(\hat{\sigma}_{\mathrm{NTNOC}}\left(\theta ; \zeta^{k}\right)\right)\right)$. Then, at the $20 \%$ perturbation strength level we found a Wilcoxon statistic of 13023.5 corresponding to a $p$-value on the order of $10^{-8}$, and at the $25 \%$ perturbation strength level we found a Wilcoxon statistic of 14593.5 corresponding to a $p$-value on the order of $10^{-9}$. In these two cases we reject the null hypothesis and conclude the reinforcement learning agent produces chemotherapeutic schedules with higher median scaled score on perturbed patients than the NTNOC. In this situation, the nearest training neighbour optimal controller is able to produce schedules more competitive with the reinforcement learning agent than those produced by the nominal optimal controller. In the $15 \%$ case, the NTNOC outperforms the reinforcement learning agent by a small margin (difference in median scores of 0.003 in favour of the NTNOC) whereas the reinforcement learner outperforms the NTNOC in the $20 \%$ case (difference in median scores of 0.077 in favour of the reinforcement learner) and the $25 \%$ case (difference in median scores of 0.060 in favour of the reinforcement learner). While the NTNOC produces more robust schedules for small perturbations, such schedules seem to only slightly outperform the schedules produced by the reinforcement learning agent. In contrast, for medium perturbations around $20 \%$ and $25 \%$, the reinforcement learning agent outperforms the NTNOC.

In Fig. 10 we concern ourselves with, once again, examining the histograms of the scores of these two agents. As before, we note that the reinforcement learner derived schedules are robust to these perturbations in patient specific parameter values, which is the source of the success in Fig. 9b, c. However, in contrast to Fig. 8, we note that the nearest training neighbour optimal controller produces schedules whose scores produce a histogram that is less diffuse than that produced by the nominal optimal controller (standard deviations of $(0.007,0.06,0.06)$ for the nearest training neighbour optimal controller at the $15 \%, 20 \%$, and $25 \%$ perturbation strength compared to standard deviations of $(0.09,0.14,0.16)$ for the nominal optimal controller and $(0.01,0.01,0.01)$ for the reinforcement learning agent). The end result is, by allowing the optimal controller access to more distribution-level data, it is capable of customizing schedules in a way that is more robust to perturbations in the patient specific 


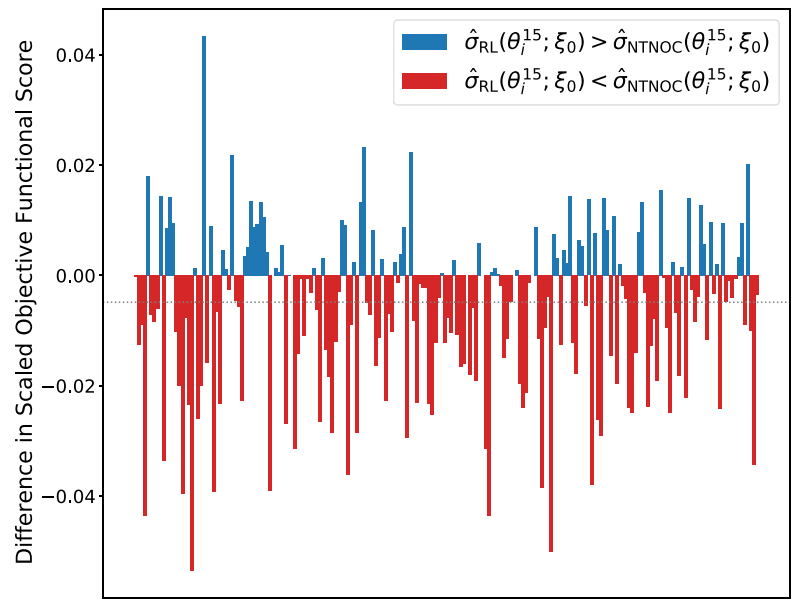

(a) Difference in scaled objective functional score for virtual patients at $15 \%$ perturbation strength.

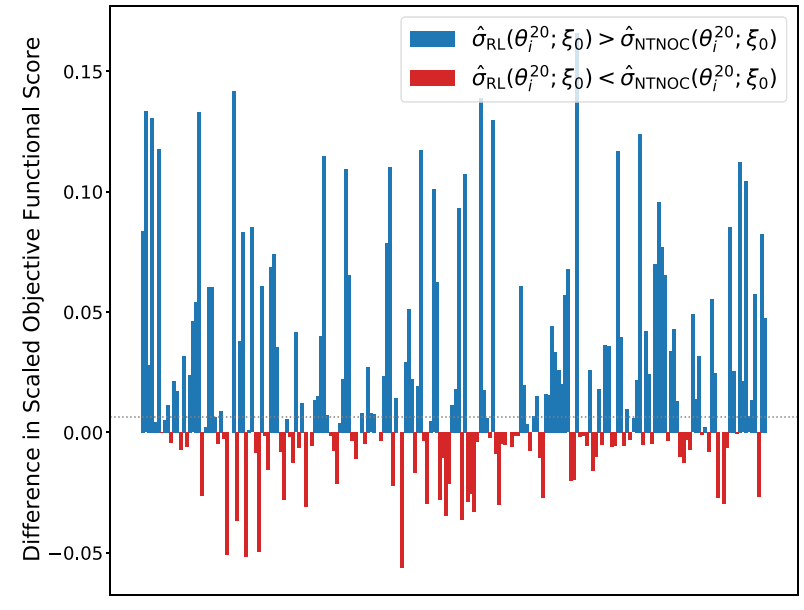

(b) Difference in scaled objective functional score for virtual patients at $20 \%$ perturbation strength..

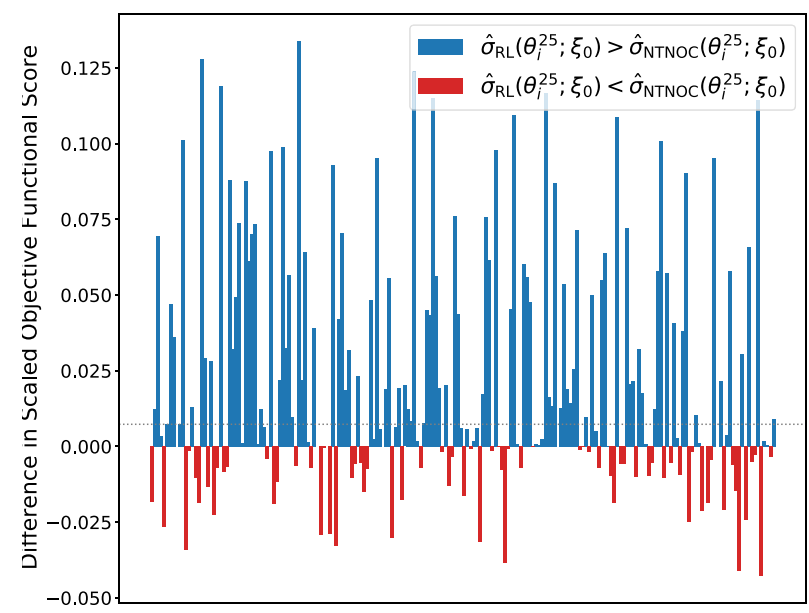

(c) Difference in scaled objective functional score for virtual patients at $25 \%$ perturbation strength.

Figure 9. Bar plots of the difference between the scores obtained by the reinforcement learner derived policy and the scores obtained by the nearest training neighbour optimal controller on all test virtual patients (i.e. bar plots of $\left.\hat{\sigma}_{\mathrm{RL}}\left(\theta_{i}^{k} ; \xi_{0}\right)-\hat{\sigma}_{\mathrm{NTNOC}}\left(\theta_{i}^{k} ; \zeta^{k}\right)\right)$. Testing patients where the reinforcement learner outperformed the optimal controller are marked in blue and patients where the optimal controller outperformed the reinforcement learner are marked in red. The dotted grey lines in each plot indicate difference in the median values of the scores obtained by the reinforcement learning agent and those obtained by the nearest training neighbour optimal controller.

parameters. However, for sufficiently high perturbations in the strength of the parameters, these personalized schedules are still less optimal than the personalized schedules produced by the reinforcement learning agent. Again, we note that the success of the reinforcement learning agent is due to the increased diffusivity of the distribution of scores obtained by the NTNOC as perturbation strength increases as contrasted with the more stable distribution of reinforcement learning agent derived scores under the same perturbation strengths.

\section{Discussion}

In summary, we examined a model of breast cancer, ovarian cancer, and bone marrow density under treatment by a chemotherapeutic for which the continuous time optimal control has been analytically derived. We discretized the optimal control problem of chemotherapeutic dosing schedule under the objective functional in Eq. (2) to apply different doses every day with dose strength discretized to be in 0 to 1 inclusive by steps of size 0.1 . By solving this discretized problem on 200 testing virtual patients, we were able to establish ground truth levels for theoretical maximal objective functional scores. We then contrasted a reinforcement learning agent trained on the nominal parameter set with a traditional optimal controller on the nominal parameter set. We noted that since the reinforcement learning agent trains a fixed policy, it can customize the corresponding dose schedule to testing virtual patients, even when the patient-specific parameterization of the differential equation environment from Eq. (1) is unknown, by leveraging additional data that is easier in practice to collect. In this 


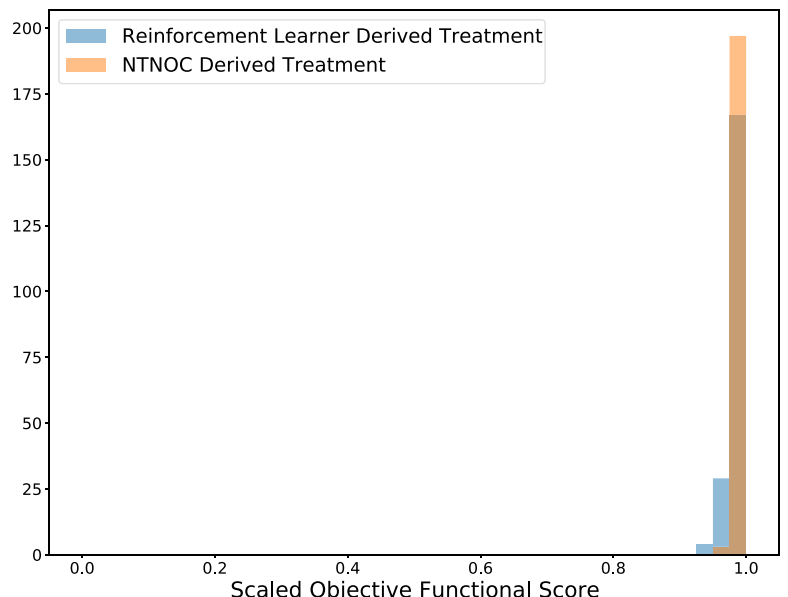

(a) At $15 \%$ perturbation strength the reinforcement agent produces a schedule that produces median scaled scores of 0.991 while the optimal controller derived schedule produces median scaled scores of 0.995

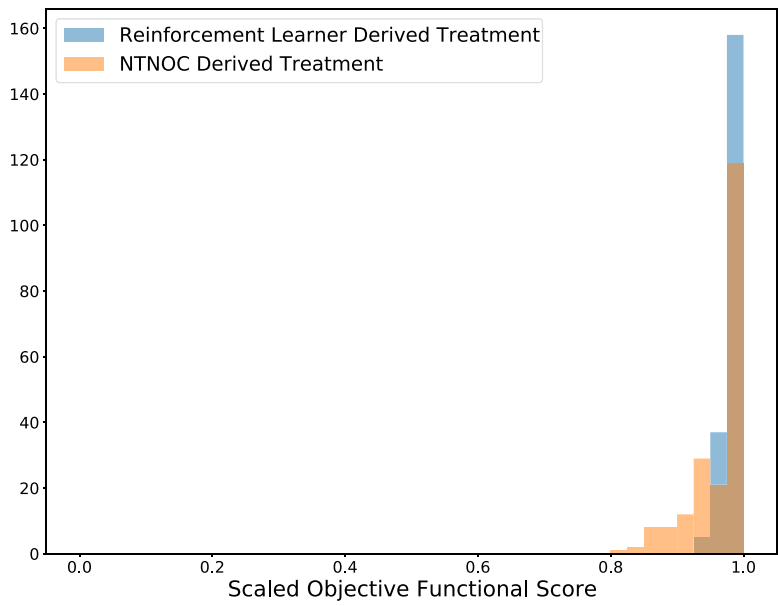

(b) At $20 \%$ perturbation strength the reinforcement agent produces a schedule that produces median scaled scores of 0.992 while the optimal controller derived schedule produces median scaled scores of 0.916 .

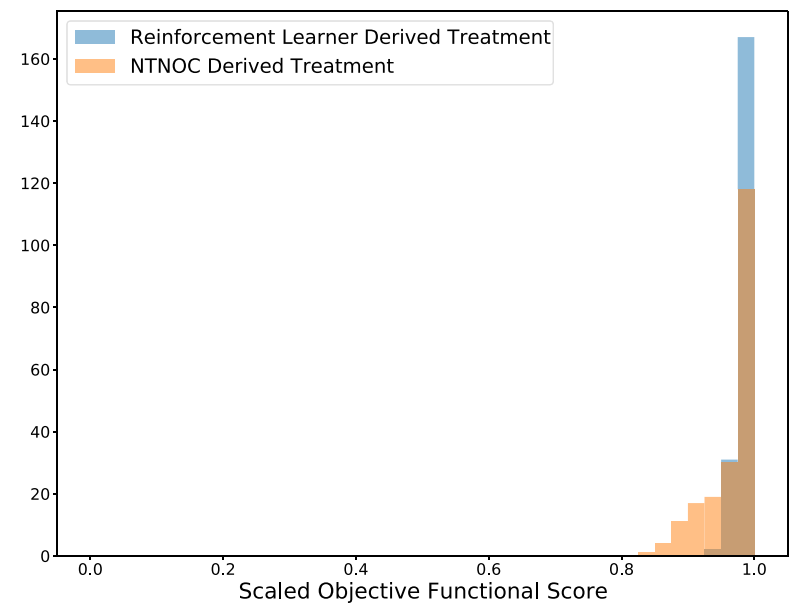

(c) At $25 \%$ perturbation strength the reinforcement agent produces a schedule that produces median scaled scores of 0.991 while the optimal controller derived schedule produces median scaled scores of 0.986 .

Figure 10. Histograms of the scores achieved by the various agents on the 200 testing virtual patients. Bin sizes were chosen to correspond to 0.025 . In particular, while the reinforcement learning agent is more robust towards perturbation in parameter values at the $20 \%$ and $25 \%$ perturbation strength, the nearest neighbour optimal controller produces schedules within $5 \%$ of the theoretical maximum at the $15 \%$ perturbation level.

case, this meant providing the reinforcement learning agent with a window of relative bone marrow density mass (relative to before the treatment process began). We noted that the reinforcement learning agent produces schedules that are closer to the theoretical optimum in a mean sense when measured against unknown patients who differ from the nominal parameter set by $15 \%, 20 \%$, and $25 \%$. In particular, we note that as the strength of perturbation increases, the net benefit of using the reinforcement learning derived schedules also increases. Moreover, as the perturbation strength increases, the collection of scaled optimality scores stay clustered between 0.925 and 1 . In contrast, as the perturbation strength increases, the collection of scaled optimality scores for the optimal controller become more diffuse.

We also allowed the optimal controller derived schedules to leverage the longitudinal relative bone marrow density information by training 1000 such optimal controllers on perturbed parameter values that were treated as known. When we compared this nearest training neighbour agent to the reinforcement learning agent, we discovered that the reinforcement learning agent still outperformed the other agent at the $20 \%$ and $25 \%$ perturbation strength level, but at the $15 \%$ perturbation strength level the nearest training neighbour agent was more optimal. However, this nearest training neighbour optimal controller was still prone to reduced performance level and a more diffuse histogram of dose-schedule scores at the higher perturbation levels, something that we did not observe in the reinforcement learning agent. 
We conclude by noting that reinforcement learning provides an agent that can be used to personalize dosage schedules in the absence of patient specific parameter data in a manner that is not prone to wild fluctuations (as evidenced by the tight histograms of Figs. 8 and 10) and did so by only requiring the mean values of the patient specific parameter distributions. In contrast, an optimal control derived agent could be improved to allow personalization of dosing schedule as well, but at the cost of requiring more samples from these patient specific distributions and the end result was still prone to fluctuations in schedule optimality.

While this particular study was focused on a situation where little patient data was used (outside of the data used to determine the nominal parameters from Table 1) one could also extend this work by allowing a reinforcement learner to learn directly from patient data, since the environment a reinforcement learning agent interacts with is effectively a black box. This method described is general and can be used for optimizing schedules for other treatments as well (i.e. radiotherapy fractions or immunotherapy treatment schedules). Moreover, this study was focused on a particular model in a mathematical oncology context, however we believe this result can be applied to other mathematical models used in cancer research and can also be extended easily to other mathematical biology contexts, or into any context wherein one needs to create a control for a system where high level distribution information about the parameters is known, but particular parameter values are unknown or prohibitively difficult to ascertain.

Received: 10 May 2021; Accepted: 9 August 2021

Published online: 09 September 2021

\section{References}

1. Panetta, J. C. \& Fister, K. R. Optimal control applied to cell-cycle-specific cancer chemotherapy. SIAM J. Appl. Math. 60, 1059-1072 (2000).

2. Yauney, G. \& Shah, P. Reinforcement learning with action-derived rewards for chemotherapy and clinical trial dosing regimen selection. In Machine Learning for Healthcare Conference, 161-226 (2018).

3. Jarrett, A. M. et al. Optimal control theory for personalized therapeutic regimens in oncology: background, history, challenges, and opportunities. J. Clin. Med. 9, 1314 (2020).

4. Rao, A. V. A survey of numerical methods for optimal control. Adv. Astronaut. Sci. 135, 497-528 (2009).

5. Beal, L. D., Hill, D. C., Martin, R. A. \& Hedengren, J. D. Gekko optimization suite. Processes 6, 106 (2018).

6. Van Hasselt, H., Guez, A. \& Silver, D. Deep reinforcement learning with double q-learning. In Proceedings of the AAAI conference on artificial intelligence, vol. 30 (2016).

7. Tesauro, G. Temporal difference learning and td-gammon. Commun. ACM 38, 58-68 (1995).

8. Bellemare, M. G., Naddaf, Y., Veness, J. \& Bowling, M. The arcade learning environment: an evaluation platform for general agents. J. Artif. Intell. Res. 47, 253-279 (2013).

9. Otto, F. Model-free deep reinforcement learning-algorithms and applications. In Reinforcement Learning Algorithms: Analysis and Applications, 109-121 (Springer, 2021).

10. Panetta, J. C. \& Adam, J. A mathematical model of cycle-specific chemotherapy. Math. Comput. Model. 22, 67-82 (1995).

11. Panetta, J. C. A mathematical model of breast and ovarian cancer treated with paclitaxel. Math. Biosci. 146, 89-113 (1997).

12. Eisen, M. Mathematical models in cell biology and cancer chemotherapy, vol. 30 (Springer, 2013).

13. Engelhardt, D. Dynamic control of stochastic evolution: a deep reinforcement learning approach to adaptively targeting emergent drug resistance. J. Mach. Learn. Res. 21, 1-30 (2020).

14. Lillicrap, T. P. et al. Continuous control with deep reinforcement learning. arXiv preprint arXiv:1509.02971 (2015).

15. Sutton, R. S. \& Barto, A. G. Reinforcement learning: an introduction (MIT press, 2018).

16. Kleijnen, J. P. An overview of the design and analysis of simulation experiments for sensitivity analysis. Eur. J. Oper. Res. 164, 287-300 (2005).

17. Mnih, V. et al. Playing atari with deep reinforcement learning. arXiv preprint arXiv:1312.5602 (2013).

18. Hedengren, J., Mojica, J., Cole, W. \& Edgar, T. Apopt: Minlp solver for differential and algebraic systems with benchmark testing. In Proceedings of the INFORMS National Meeting, Phoenix, AZ, USA, 1417, 47 (2012).

19. Hodges, J. L. The significance probability of the smirnov two-sample test. Ark. Mat. 3, 469-486 (1958).

20. Wilcoxon, F. Individual comparisons by ranking methods. Biometrics Bull. 1, 80-83 (1945).

\section{Acknowledgements}

This work was supported by the Canadian Institutes of Health Research (CIHR).

\section{Author contributions}

B.E. and M.P. conceived the experiments, B.E. conducted the experiments, B.E. analysed the results. All authors reviewed the manuscript.

\section{Competing interests}

The authors declare no competing interests.

\section{Additional information}

Supplementary Information The online version contains supplementary material available at https://doi.org/ 10.1038/s41598-021-97028-6.

Correspondence and requests for materials should be addressed to B.E.

Reprints and permissions information is available at www.nature.com/reprints.

Publisher's note Springer Nature remains neutral with regard to jurisdictional claims in published maps and institutional affiliations. 
(c) (i) Open Access This article is licensed under a Creative Commons Attribution 4.0 International cc) License, which permits use, sharing, adaptation, distribution and reproduction in any medium or format, as long as you give appropriate credit to the original author(s) and the source, provide a link to the Creative Commons licence, and indicate if changes were made. The images or other third party material in this article are included in the article's Creative Commons licence, unless indicated otherwise in a credit line to the material. If material is not included in the article's Creative Commons licence and your intended use is not permitted by statutory regulation or exceeds the permitted use, you will need to obtain permission directly from the copyright holder. To view a copy of this licence, visit http://creativecommons.org/licenses/by/4.0/.

(C) The Author(s) 2021 